\title{
Flipped Classroom en prácticas de ciencias de la salud
}

\section{Isabel Torres-Cuevas ${ }^{\mathrm{a}}$, Esther García Miralles ${ }^{\mathrm{b}}$, Marta Ribelles Llop ${ }^{\mathrm{c}}$ Laura Marqués Martínez ${ }^{\mathrm{d}}$}

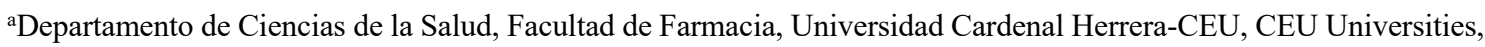
misabel.torres@uchceu.es. ${ }^{b}$ Departamento de Ciencias de la Salud, Facultad de Odontología, Universidad Cardenal

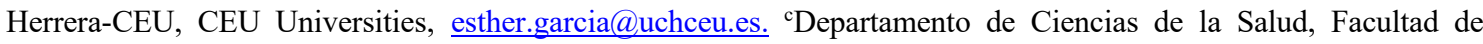
Odontología, Universidad Cardenal Herrera-CEU, CEU Universities, marta.ribelles@uchceu.es. ${ }^{\mathrm{d}}$ Departamento de Ciencias de la Salud, Facultad de Odontología, Universidad Cardenal Herrera-CEU, CEU Universities, laura.marques@uchceu.es.

\section{Abstract}

New pedagogical methodologies in the health sciences area could be necessary for trainees. Flipped Classroom or inverted classroom (FC) is a tool that offers greater autonomy for the student, as well as better knowledge assimilation. The main objective of this study was the achievement of basic skills for the application of knowledge, communication, and interpretation of relevant data to make judgments for health sciences students. The number of students participating in the study was 1103 (n=1103), all of them from different degrees such as dentistry, pharmacy, and nursing. Students were distributed in 2 study groups: Group A, application of masterclass methodologies (M), and Group B, Flipped Classroom (FC) or inverted classroom method. At the end of the practical sessions, a test was used to evaluate the students' knowledge acquisition and their degree of satisfaction with the teaching methodology. The obtained results showed a significant increase in the maximum number of correctly-answered questions and a greater degree of satisfaction from those students who attended the practical lessons taught using the FC method. As a conclusion, the application of the FC method in health sciences leads to better results in the acquisition of the course competencies than the masterclass methodologies.

Keywords: Flipped classroom, Flipped learning, Health professions education, active learning, critical thinking.

\section{Resumen}

La introducción de nuevas metodologías pedagógicas en el ámbito de ciencias de la salud es una herramienta necesaria para los alumnos en formación. Flipped Classroom o aula invertida (FC) es una herramienta que proporciona una mayor autonomía para el alumno, así como una mayor asimilación de conocimientos. El objetivo principal del estudio fue la adquisición de competencias básicas de aplicación de conocimientos, comunicación e interpretación de datos relevantes para emitir juicios en estudiantes de ciencias de la salud. Los participantes en el estudio fueron $n=1103$ estudiantes de odontología, farmacia y enfermería, los cuales se dividieron en 2 grupos de estudio; 
Grupo A, aplicación de metodologías de clases magistrales (M) y Grupo B aplicación aula invertida "Flipped Classroom" (FC). Al final de las prácticas se realizó un cuestionario para la evaluación de la adquisición de conocimientos y el grado de satisfacción. Los resultados obtenidos demostraron un aumento significativo en el máximo número de preguntas correctamente contestadas (FC) y un mayor grado de satisfacción de los alumnos que realizaron las prácticas mediante FC. Como conlusión podríamos indicar que la aplicación de FC en ciencias de la salud presenta mejores resultados en la adquisición de conocimientos que las metodologías didácticas magistrales.

Palabras clave: aula invertida, aprendizaje invertido, educación profesionales de la salud, aprendizaje activo, evaluación, pensamiento crítico, metacognición.

\section{1.-Introducción}

Los profesionales de la salud deben tener un aprendizaje global. Además de saber aplicar conceptos básicos a la práctica clínica deben adquirir nuevas competencias específicas e interpersonales que faciliten la interacción social y la cooperación (Carey, 2010). En la actualidad se tiene en gran valor aquellos programas universitarios que incluyen oportunidades para que los alumnos trabajen en entornos de aprendizaje activos, de modo que puedan desarrollar habilidades de aprendizaje permanente y competencias relacionadas con la profesionalidad, la ética médica, las humanidades y la colaboración interprofesional (Ramnanan, 2017).

Debido a estas consideraciones muchos educadores que trabajan impartiendo docencia con alumnos de grado de ciencias de la salud están aplicando el aula invertida a su enseñanza (Ramnanan, 2017; Hew, 2018).

El Flipped Classroom (FC) o aula invertida es un modelo pedagógico que transfiere el trabajo de determinados procesos de aprendizaje fuera del aula y utiliza el tiempo de clase, junto con la experiencia del docente, para facilitar y potenciar otros procesos de adquisición y práctica de conocimientos dentro del aula (Lowell, 2013; Buhr, 2014; Alvarez, 2018).

En el modelo de aula tradicional, los estudiantes se exponen primero al contenido educativo a través de conferencias didácticas impartidas por el profesor y posteriormente el aprendizaje es reforzado por trabajo en clase, donde los alumnos tienen la oportunidad de aplicar los conocimientos recién adquiridos. Por el contrario en el modelo FC los alumnos se exponen primero al contenido educativo antes de las sesiones de clase a través de lecturas, vídeos u otros ejercicios que les facilita el profesor, adquiriendo unos conocimientos previos. A continuación, el tiempo en el aula se dedica a aplicar y afianzar sus conocimientos en un entorno que promueve la colaboración son sus compañeros y la retroalimentación. Al proporcionar a los estudiantes contenido fundamental clave antes de la clase les permite a los alumnos participar en el contenido a su propio ritmo, controlando cuándo y cuánto lo ven, proporcionando la base para el aprendizaje aplicado en clase, que luego se refuerza a través del trabajo y la exploración (Persky, 2017; Ramnanan, 2017).

$\mathrm{Al}$ analizar la percepción del FC en estudiantes de ciencias de la salud se observó una gran satisfacción con las primeras aplicaciones y generalmente prefirieron este método a la enseñanza tradicional. Al mismo tiempo se produjo una mejora significativa en el aprendizaje de los estudiantes en comparación con los métodos de enseñanza tradicionales (Ramnanan, 2017; Hew, 2018). 
El aula invertida representa un avance importante en la educación de profesinales de la salud. Sin embargo, queda por ver si estos modelos pueden ser aplicados a todos los estudiantes independientemente de su contexto geográfico.

\section{2.- Objetivos}

\subsection{Objetivo general}

El objetivo general fue analizar la adquisición de competencias básicas de aplicación de conocimientos, comunicación e interpretación de datos relevantes para emitir juicios en estudiantes de ciencias de la salud.

\subsection{Objetivos específicos:}

1. Estudio del grado de satisfacción de los alumnos comparando las prácticas realizadas con Flipped Classroom $V s$ las clases magistrales.

2. Análisis global de la aplicación del aula invertida en prácticas de ciencias de la salud.

3. Comparar la adquisición de conocimientos de los alumnos con la aplicación del Flipped Clasroom $V s$ clase magistral en los diferentes grados de odontología, enfermería y farmacia.

\section{3.- Desarrollo de la innovación}

El proyecto se realizó con alumnos del grado de enfermería y farmacia matriculados en el primer curso en la asignatura de estructura y función del cuerpo humano I y alumnos de $4^{\circ}$ y $5^{\circ}$ curso del grado de odontología matriculados en odontopediatría y prácticum infantil I. El número total de alumnos participantes fue de 1103. En el grado de enfermería participaron 399, en el de farmacia 64 y en el de odontología 640 (232 alumnos de $4^{\circ}$ curso y 408 de $5^{\circ}$ curso).

Respecto al porcentaje total de alumnos participantes en el estudio fue un $69 \%$ mujeres frente al $31 \%$ que fueron hombres. En el estudio el $49 \%$ de los alumnos realizaron las prácticas bajo las condiciones del método Flipped Classroom (FC) y el 51\% del método Magistral (M). Además, el rango de edades del estudio fue desde los 18 hasta mayores o igual de 25 años, siendo los mayores porcentajes para los mayores o igual de 25 años con el $24 \%$ de los casos seguido de 22 años con un $14 \%$. El resto de porcentajes los podemos observar en la Figura 1. 


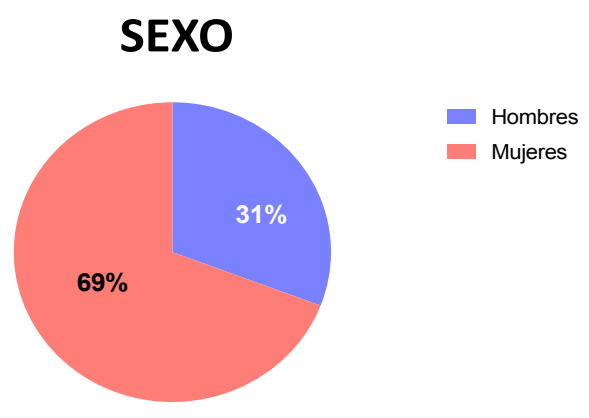

\section{EDAD}

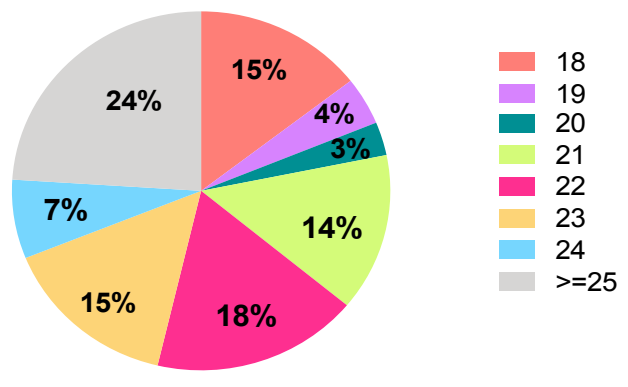

MÉTODO

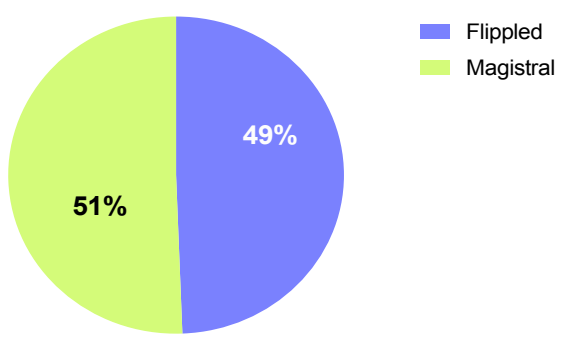

Fig 1. Representación porcentual de los parámetros sexo, método y edad del estudio.

Para la realización del estudio los alumnos participantes fueron divididos de manera aleatoria mediante un programa estadístico (Epidat 3.1, Xunta de Galicia, Spain) en diferentes grupos de estudio, concretamente en 6 grupos:

\section{$\checkmark$ GRUPO A ENFERMERIA \\ $\checkmark$ GRUPO B ENFERMERIA \\ $\checkmark$ GRUPO A FARMACIA \\ $\checkmark$ GRUPO B FARMACIA \\ $\checkmark$ GRUPO A ODONTOLOGÍA \\ $\checkmark$ GRUPO B ODONTOLOGÍA}

Todos los grupos A realizaron una actividad docente basada en metodologías didácticas tradicionales o clases magistrales (M). Antes de la realización de la práctica el profesor facilitó a todos los alumnos el contenido educativo para su lectura y comprensión de manera individual, adquiriendo unos conocimientos previos.

Los alumnos asistieron a las prácticas donde en primer lugar el profesor realizó una clase magistral a través de una conferencia didáctica sobre el contenido de la práctica y a continuación los alumnos realizaron dicha actividad, poniendo en práctica los conocimientos recién adquiridos.

Todos los grupos B realizaron una actividad docente basada en el aula invertida "Flipped Classroom" (FC). Los alumnos se dividieron en 4 subgrupos de forma aleatoria, los grupos de expertos. La asignación tuvo en cuenta factores que promovieron la integración de los diferentes estudiantes desde un punto de vista 
social y cultural (estudiantes extranjeros con españoles o estudiantes de diferentes ciudades con los de Valencia).

Antes de la realización de la práctica el profesor facilitó a los grupos de expertos el contenido educativo para su lectura, comprensión y preparación de la exposición que realizaron a sus compañeros. En cada práctica el grupo de expertos correspondiente realizó la exposición a sus compañeros y les ayudó a aplicar y afianzar sus conocimientos mientras realizaban la práctica, resolviendo las dudas que tuvieron. El profesor durante la práctica tenía como función alentar a los estudiantes en la investigación individual y el esfuerzo colaborativo.

Tabla 1. Descripción de las actividades de aprendizaje en los grupos de estudio.

\begin{tabular}{|c|c|c|}
\hline & ANTES DE LA CLASE & DURANTE LA CLASE \\
\hline $\begin{array}{c}\text { GRUPO A } \\
\text { METODOLOGÍA } \\
\text { TRADICIONAL }\end{array}$ & - Lectu & $\begin{array}{l}\text { - Clase magistral de los contenidos } \\
\text { teóricos por parte del profesor } \\
\text { - Realización de la práctica }\end{array}$ \\
\hline $\begin{array}{c}\text { GRUPO B } \\
\text { FLIPPED } \\
\text { CLASSROOM }\end{array}$ & $\begin{array}{l}\text { - } \quad \text { Constitución grupos de expertos } \\
\text { - Trabajo del grupo de expertos en el } \\
\text { tema asignado } \\
\text { - } \quad \text { Lectura del contenido educativo }\end{array}$ & $\begin{array}{l}\text { - Exposición del grupo de expertos de los } \\
\text { contenidos teóricos } \\
\text { - Realización de la práctica }\end{array}$ \\
\hline
\end{tabular}

Todos los alumnos de todos los grupos al finalizar la práctica realizaran un cuestionario anónimo, en el cual quedó reflejado el grupo de prácticas al que pertenecían, la edad y el sexo de los alumnos. Este cuestionario constó de dos apartados: El primero sirvió para evaluar el grado de satisfacción del alumno con las prácticas a través de una valoración de 0 (muy insatisfecho) al 5 (excelente) como se muestra en la Figura 2, donde se valoraron los objetivos, los contenidos, la metodología, la explicación en el aula y la calidad y cantidad de los documentos. El resultado de esta parte se mostró gráficamente con el valor de 0 a 5 obtenido para cada uno de los ítems en referencia a cada uno de los métodos aplicados (clase magistral Vs Flipped Classroom). Por otro lado, se montró una representación de las edades y sexo de los participantes.

El segundo apartado contó de 4 preguntas relacionadas con el contenido teórico-práctico de la práctica, el cual sirvió para evaluar la adquisición de conocimientos. Para hallar los resultados se tuvo en cuenta el porcentaje (\%) de alumnos que respondieron correctamente a cada pregunta. Es decir, el \% de alumnos que respondieron bien a $0,1,2,3$ o 4 preguntas y se compararon ambos métodos, realizando la estadística adecuada. 


\section{PROYECTO DE INNOVACION Y MEJORA DE LA DOCENCIA}

1. SEXO:

MASCUUINO

FEMENINO

2. EDAD

3. GRUPO

4. Valora las siguientes preguntas del 0 al 5 siendo 5 excelente y 0 muy insatisfecho.

\begin{tabular}{|l|l|l|l|l|l|l|}
\hline & 5 & 4 & 3 & 2 & 1 & 0 \\
\hline Los objetivos de la práctica se han conseguido & & & & & & \\
\hline $\begin{array}{l}\text { El contenido de la practica ha satisfecho mis necesidades de } \\
\text { formación }\end{array}$ & & & & & & \\
\hline $\begin{array}{l}\text { La metodología usada ha sido la más adecuada a los objetivos y } \\
\text { contenidos }\end{array}$ & & & & & & \\
\hline La metodología ha permitido una activa participación & & & & & & \\
\hline Las explicaciones y ejercicios han sido útiles y suficientes & & & & & \\
\hline La calidad y cantidad de la documentación han sido adecuadas & & & & & & \\
\hline
\end{tabular}

Fig 2. Imagen de la primera parte del cuestionario

El análisis estadístico que se empleó para el análisis de variables cualitativas fue el test Chi cuadrado. Se utilizó una P de 0,05 como límite para la aceptación de diferencias estadísticamente significativas. El manejo de los resultados se realizó con la herramienta estadística del programa informático GhaphPad Prism 8 (GraphPad Software Inc., California, EEUU).

\section{4.- Resultados}

En referencia al grado de satifacción de los alumnos frente a las diferentes metodologías utilizadas, se valoraron los objetivos, el contenido de la práctica, la metodología empleada, la utilidad que podría tener la práctica para su formación (como complemento a la parte teórica de la asignatura), la participación de los alumnos y la calidad. Los resultados mostraron un aumento significativo en cada uno de los ítems: objetivos, contenido, metodología y utilidad del grado de satisfacción \% (excelente) para la metodología FC frente a M como se observa en la Figura 3. En esta figura no se muestran los valores del 0 al 2 porque en ninguno de los casos habían alumnos que hubieran contestado esta valoración. 
Objetivos

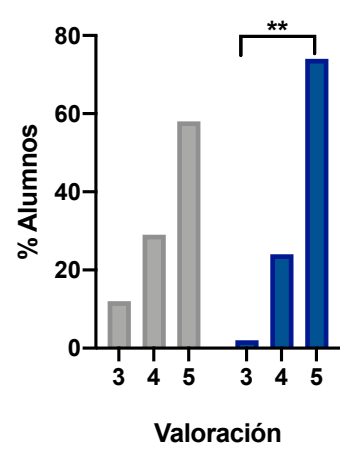

Participación

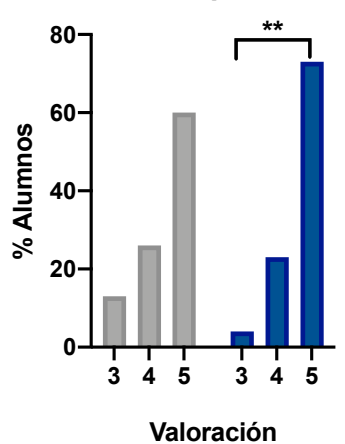

Contenido

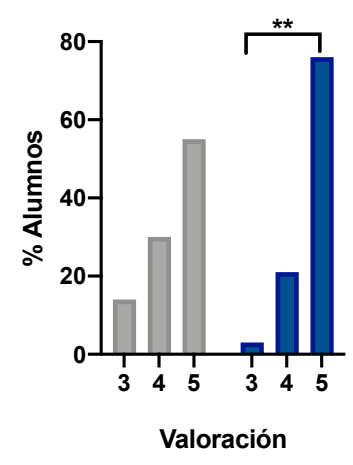

Metodología

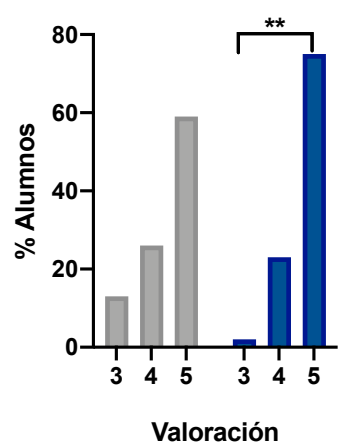

Utilidad

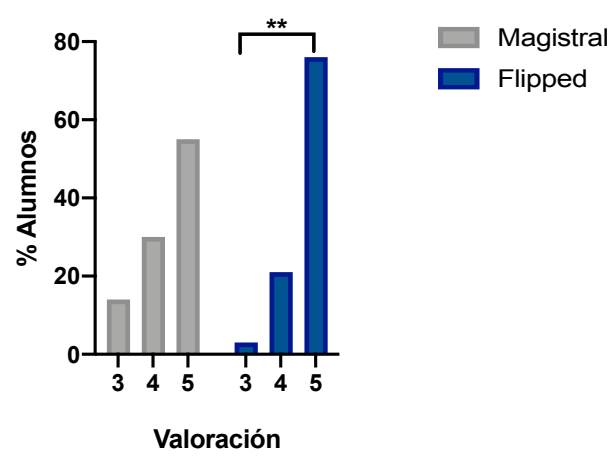

Calidad

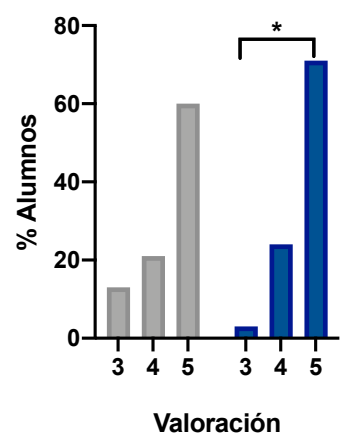

Fig3. Porcentaje de número de alumnos que han valorado los objetivos, contenido, utilidad, participación, metodología y calidad en cada uno de los métodos de estudio, Magistral (M) y Flipped Classroom (FC). La diferencia estadística se indica como sigue * $<0.05$ VS $M, * *<0.001 V_{S} M$.

Los resultados de la aplicación del aula invertida o Flipped classroom (FC) frente a las clases magistrales (M) dieron resultados satisfactorios en referencia al grado de adquisición de conocimientos. El resultado global, es decir, de todas las preguntas realizadas a los alumnos de los diferentes grados y del total de todas la prácticas con un valor de $\mathrm{n}=1103$, muestra un aumento significativo en el $\mathrm{FC}$ respecto a $\mathrm{M}$ en el mayor número de preguntas correctas, es decir, 4. En cuanto al número de aciertos igual al 3, los resultados no muestran diferencias. Para un aprobado, que sería un número de aciertos igual a dos, se obtiene una disminución significativa en el grupo $\mathrm{FC}$ al igual que para número de aciertos 1 . En el caso de los alumnos que no han acertado ninguna de las preguntas, no observamos diferencias estadísticamente significativas (Figura 4.A).

Si pasamos al análisis por cada uno de los grados en los que se ha realizado el estudio, en el grado de enfermería no se observan diferencias significaticas, pero sí una tendencia a un mayor porcentaje de alumnos que responden mayor número de respuestas, es decir, 3 y 4 correctas en el método FC comparado con el $\mathrm{M}$ con un valor de n=399 (Figura 4.B). En el grado de farmacia se observa similar al de enfermería, en este caso el aumento es significativo para el numero de aciertos 4 con el método FC. Además se puede observar que no hay ningún alumno en el número de respuestas 0,1 y 2 versus al método $\mathrm{M}$ (Figura 4.B). El número de alumnos participantes es $n=64$. Por último, en el grado de odontología, si diferenciamos por 
cursos, $\operatorname{los}$ de $4^{\circ}$ curso $(n=232)$ muestran prácticamente igual $\%$ de alumnos que han acertado correctamente el mismo número de respuestas correctas, excepto en el número de acierto igual a 3 que el método $\mathrm{M}$ parece incrementar. Para los alumnos de $5^{\circ}$ curso $(\mathrm{n}=408)$, hay un claro aumento significativo para el método FC en el número de aciertos igual a 4. Hay un aumento significativo para el número de respuesas acertadas igual a 2 a favor del método M. Cabe destacar que no hay ningún alumno con el método FC en el número de respuestas igual a 0 y 1 (Figura 4.B).

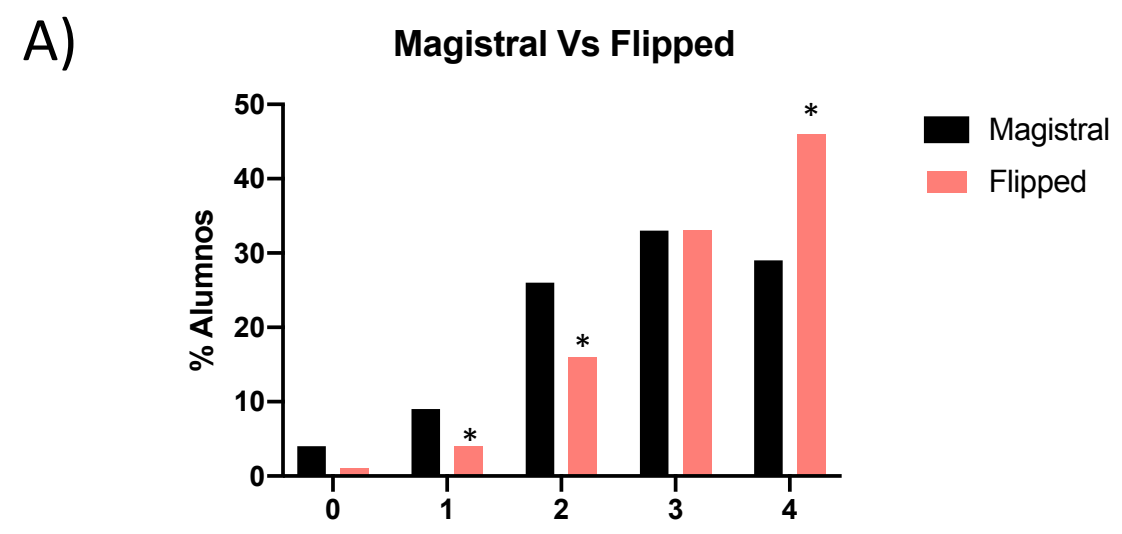

$\mathbf{N}^{\circ}$ Respuestas Acertadas

B)

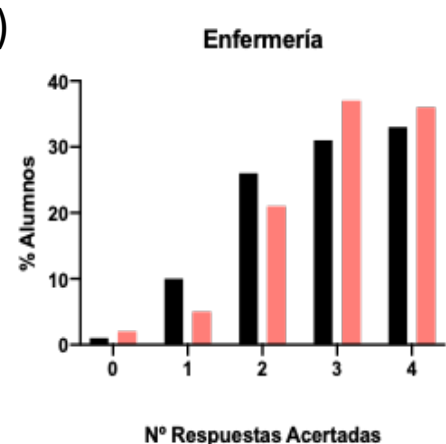

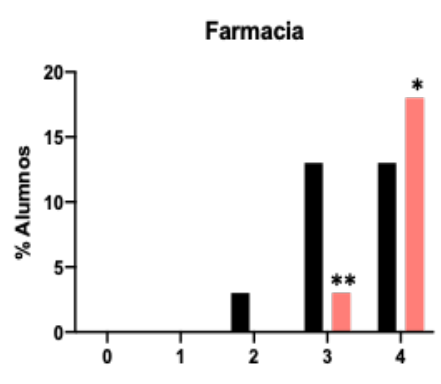

$\mathrm{N}^{\circ}$ Respuestas Acertadas

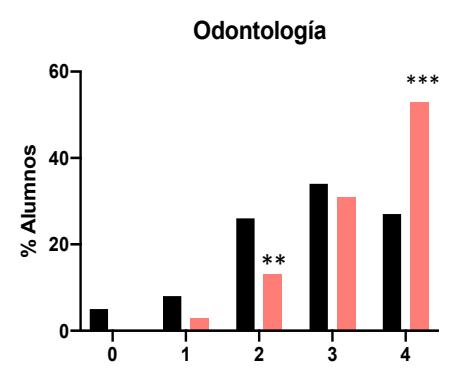

$\mathbf{N}^{\circ}$ Respuestas Acertadas

Fig4. A)Porcentaje de número de alumnos total y B) porcentaje de cada uno de los grados (enfermería, farmacia y odontología) en función del número de respuetas acertadas en función de la metodología empleada: Magistral (M) y Flipped Classroom (FC). La diferencia estadística se indica como sigue $*<0.05$ Vs M, **<0.001Vs My ***<0.0001Vs M.

\section{5.- Conclusiones}

La conclusión general es que la aplicación de la metodología Flipped Classroom (FC) en las prácticas de ciencias de la salud presenta mejores resultados en la adquisición de conocimientos que las metodologías didácticas magistrales. Además de tener muy buen grado de aceptación y aumentar la participación y motivación por parte del alumnado de los diferentes grados. Además también podemos afirmar que los alumnos lo consideran de gran utilidad como lo han reflejado en el cuestionario. 
En los grados de farmacia y odontología los resultados reflejan que la mayoría del alumnado obtiene la máxima cualificación con la metodología FC, mientras que en el grado de enfermería los alumnos que han recibido la metodología FC presentan mayor puntuación que los alumnos que han recibido las clases magistrales aunque no existen diferencias estadísticamente significativas. Estos resultados en el primer curso del grado de enfermería pueden ser debidos a la falta de cooperación y de trabajo en equipo de los alumnos hecho que limita el aprovechamiento al máximo de la metodología FC.

Por tanto, podríamos considerada una herramienta educativa eficaz en el ámbito universitario ya que la adquisición de los conocimientos es mayor con la aplicación del FC. Este nuevo tipo de metodología podría estar ayudando a afianzar los conocimientos teóricos a través de las clases prácticas.

Este estudio nos abre una línea futura de actuación diferente a la hora de realizar nuestra docencia práctica y quizás podría ser ampliado en un futuro no solo en las prácticas de los grados sino también ser otra herramienta metodológica para aplicar en las tutorías y comprobar si podría ayudar al entendimiento de los conceptos teóricos a través de los exámenes, como hemos demostrado en las prácticas.

\section{6.- Referencias}

1. ALVAREZ AM (2018). "Aula invertida en odontología” en Acta Odontologica Venezolana; 56.

2. BUNH GT, HEFLIN MT, WHITE HK, PINHEIRO SO (2014). "Using the jigsaw cooperative learning method to teach medical students about long-term and postacute care" en Journal of the American Medical Directors Association, 15, p. 429-34.

3. CAREY JA, MADILL A y MANOGUE M. (2010). "Communication skills in dental education: a systematic research review" en European Journal of Dental Education, 14, p. 69-78.

4. HEW KF, LO CK (2018). "Flipped classroom improves student learning in health professions education: a meta-analysis"en BMC Med Educ,18, p. 38.

5. LOWELL J, VERLEGER MA (2013). "The Flipped Classroom: A Survey of the Research" en American Society for Engineering Education, June, p. 23-6.

6. PERSKY AM, MCLAUGHLIN JE (2017). "The flipped classroom-from theory to practice inhealth professional education” en Am J Pharm Educ, 81, p. 118.

7. RAMNANAN CJ, POUND LD (2017). "Advances in medical education and practice: student perceptions of the flipped classroom" en Adv Med Educ Pract, 8, p. 63-73. 